Scientific Visualization, 2021, volume 13, number 3, pages 115 - 124, DOI: 10.26583/sv.13.3.12

\title{
Registration of Displacement and Visualization of the Deformation Field of a Cylindrical Shell under its Shock-Wave Loading
}

\author{
S.I. Gerasimov',A,B,C,D, K.V. Totyshev²,A, N.A. Trepalov3,A, R.V. Gerasimova4,B \\ A Russian Federal Nuclear Center All-Russian Research Institute of Experimental \\ Physics, Sarov, Nizhny Novgorod region, 607188 Russia \\ B Sarov State Physics and Technical Institute of National Research Nuclear University \\ MEPhI, Sarov, Nizhny Novgorod region, 607186 Russia \\ c Nizhny Novgorod State Technical University, Nizhny Novgorod, 603950 Russia \\ D Institute for Problems of Mechanical Engineering, Institute of Applied Physics, Russian \\ Academy of Sciences, Nizhny Novgorod, 603024 Russia \\ 1 ORCID: 00oo-0002-6850-0816, s.i.gerasimov@mail.ru \\ 2 ORCID: 0000-0002-6232-505X, ktotyshev@bk.ru \\ 3 ORCID: oooo-0001-5703-9027, natrepalov@mail.ru \\ 4 ORCID: oooo-0002-2711-3975, r.v.gerasimova@mail.ru
}

\begin{abstract}
This paper discusses and demonstrates the approach to the registration of the elastic deformations and vibrations of the barrel of a light-gas gun based on high-speed video recording and the subsequent processing of the obtained images by a cross-correlation algorithm. The use of high-speed video recording of the cylindrical shell surface and crosscorrelation analysis of the obtained images allows determining the space-time dependencies of the movement of the recorded surface and visualizing the deformation field. The approach is demonstrated by the example of high-speed video recording of a fragment of the barrel of a light-gas gun LGG-5,6 in the process of firing. Vibrations of the barrel in the vertical and horizontal directions were recorded, the maximum amplitude value of which was $5 \mathrm{~mm}$. We also visualize for analysis the field of longitudinal elastic deformations of the barrel caused by the passage of the striker through the barrel channel.

Keywords: visualization, cross-correlation algorithm, deformation, elastic vibrations of the barrel, internal ballistics.
\end{abstract}

\section{Introduction}

The development of digital image recording and processing systems has significantly expanded the capabilities of optical diagnostic methods. Recently it is possible to program image processing in order to obtain quantitative information about the physical process under study. Methods based on the correlation of digital images are widely used. Initially, the digital image correlation method was used primarily for the analysis of velocity fields in the digital tracer anemometry (Particle Iimage Velocimetry - PIV) method [1]. Further, this image processing method was used to analyze images of high-speed gas-plasma and liquid flows [2], to visualize the flow structure in a water drop [3], to visualize perturbations of the sandy medium $[4,5]$, as well as to visualize displacements and deformations $[6,7]$.

One of the tasks of ballistics is to determine the elastic deformations and vibrations of the barrel during the shot. The intense increase in pressure and the accelerated movement of the projectile through the barrel channel when fired causes the barrel to oscillate in different directions [8]. The study of the dynamics of the barrel in the process of firing is an actual scientific and practical problem, since deformations determine the initial 
conditions of the projectile movement on the external ballistic trajectory, for example, the angle of departure of the projectile from the barrel channel, and, ultimately, affect the accuracy of shooting. One of the first works devoted to this topic is the monograph [9], which takes into account many factors that affect the interaction of the projectile with the barrel. The most complete statement of the problem of the oscillation of the barrels of artillery systems when fired, in the framework of a one-dimensional model of elastic dynamics, is presented in [10].

In this paper, we demonstrate the registration of the elastic deformations and vibrations of the barrel of a light-gas gun (LGG) on the basis of high-speed video recording and subsequent processing of the obtained images by a cross-correlation algorithm.

\section{Setting up the experiment}

In the experiment, high-speed video recording of the section of the LGG-5.6 barrel of the $5.6 \mathrm{~mm}$ caliber was carried out when fired. LGG-5,6 is designed for throwing simulators of small particles of" space debris "weighing up to $0.1 \mathrm{~g}$ in the speed range from 2.5 to $8 \mathrm{~km} \mathrm{/} \mathrm{s}[11,12]$. In this experiment, a spherical impactor made of an aluminum alloy weighing $0.02 \mathrm{~g}$ was thrown at a speed of $\sim 6.5 \mathrm{~km} / \mathrm{s}$. The appearance of LGG-5,6 and its scheme are shown in Figure 1.

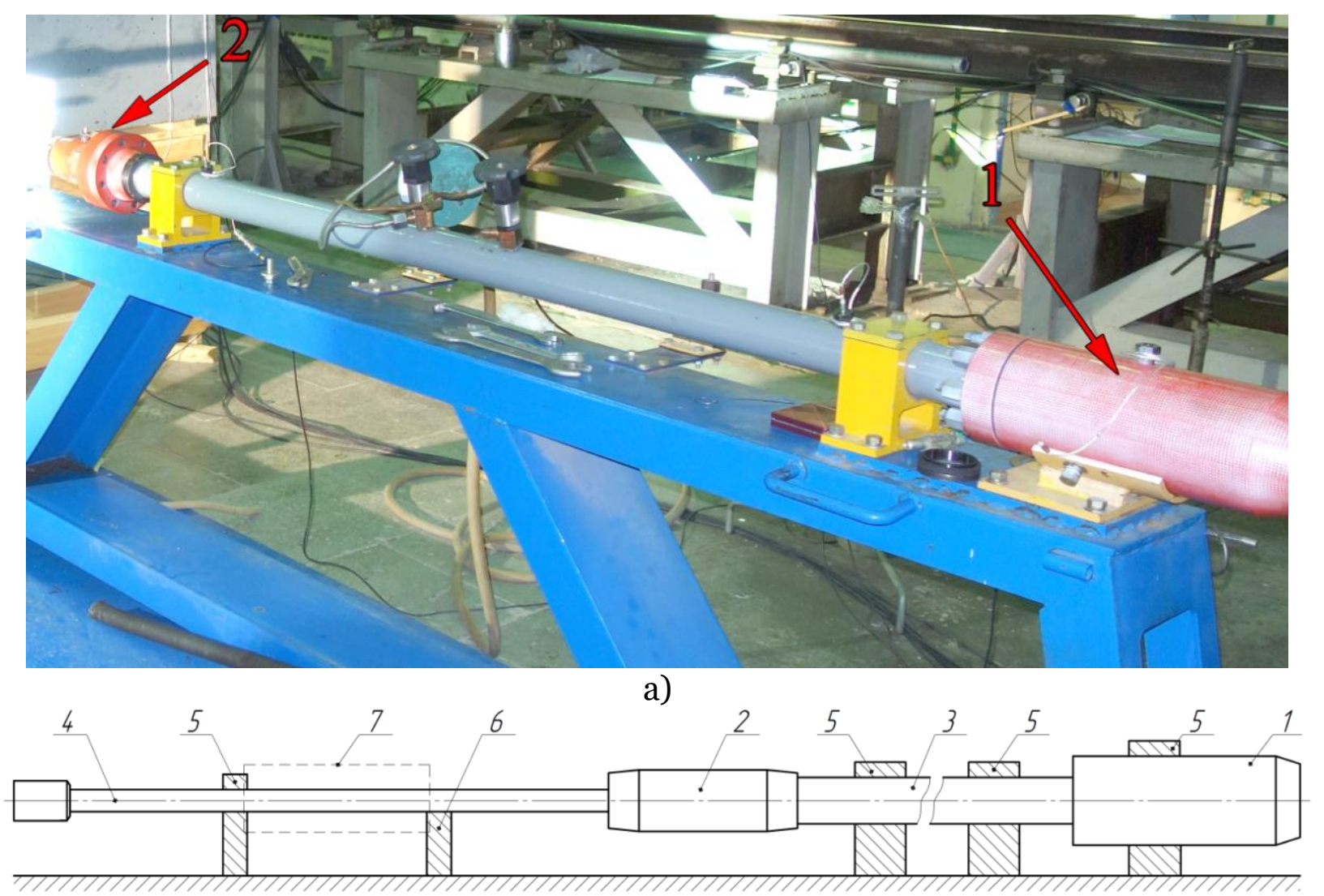

b)

Figure 1-Appearance of LGG-5,6 (a) and its diagram (b): 1-charging chamber, 2-forkamera, 3-piston barrel, 4-ballistic barrel, 5-support, 6-support, 7-video recording area.

The video recording scheme provided images of the ballistic barrel (BB) in the area from the support to the lodgment at discrete times relative to the launch of the LGG-5, 6 (the external diameter of the $\mathrm{BB}$ is $36 \mathrm{~mm}$ ). Video recording was performed under external illumination conditions with a shooting frequency of $69 \mathrm{kHz}$, a frame exposure time of 1 microsecond, and a frame size of $1280 \times 256$ pixels. A gas-discharge searchlight with an 
electric power of $700 \mathrm{~W}$ and a color temperature of $5500 \mathrm{~K}$ was used as a light source. The spatial resolution of the video recording scheme was 3.84032 pixels / $\mathrm{mm}$.

In order to determine the movement and visualization of the deformation field of the recorded part of the $\mathrm{BB}$ during the shot, a contrasting pattern was applied to its surface, representing horizontal and vertical lines with a thickness of $\sim 0.3 \mathrm{~mm}$ with a step of $\sim 4$ $\mathrm{mm}$ (Figure 2).

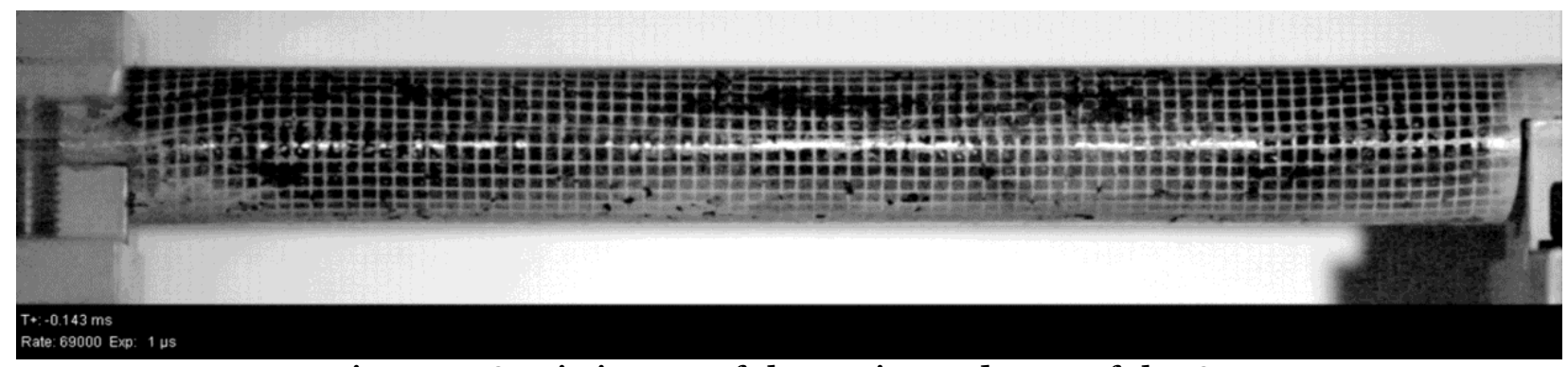

Figure 2-Static image of the registered part of the SB.

The launch of the high-speed video camera was performed from the signal to activate the LGG-5,6. The total registration time was $300 \mathrm{~ms}$. At the time of $17.641 \mathrm{~ms}$ relative to the triggering signal, a pulsed change in illumination is observed in the registration region, which is characteristic of the muzzle exhaust [13].

\section{Analysis of the results}

The recorded video information was analyzed using a cross-correlation algorithm. Firstly, the frames obtained after the triggering signal were analyzed relative to the reference (static) image of the BB. The processing was carried out in the program "PIVview2CDemo" [14]. A multi-pass cross-correlation processing algorithm with a square survey window and 50\% overlap was used. The size of the survey window was iteratively reduced from 256 to 16 pixels. The region of interest (ROI) was limited to the coordinates $(100,50)$ and $(1240,180)$ pixels. The correlation function was approximated using threepoint Gaussian interpolation with subpixel interpolation. The fast Fourier transform algorithm was used to calculate the correlation function.

Secondly, we analyse the entire array of the received images. Fragments of the processing results, in the form of a vector field of displacements of the BB surface relative to the static state, are shown in Figure 3.

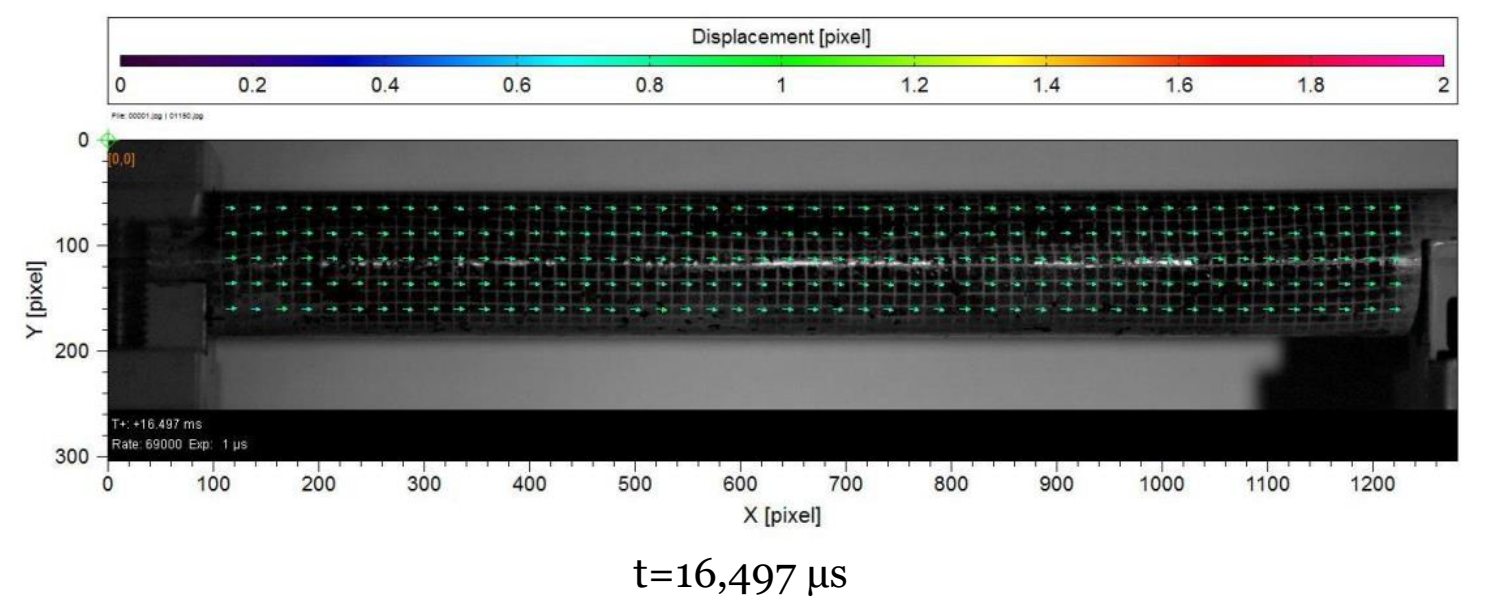




\begin{tabular}{|c|c|c|c|c|c|c|c|c|c|c|}
\hline \multicolumn{11}{|c|}{ Displacement [pixel] } \\
\hline 0 & 0.2 & 0.4 & 0.6 & 0.8 & 1 & 1.2 & 1.4 & 16 & 1.8 & 2 \\
\hline
\end{tabular}
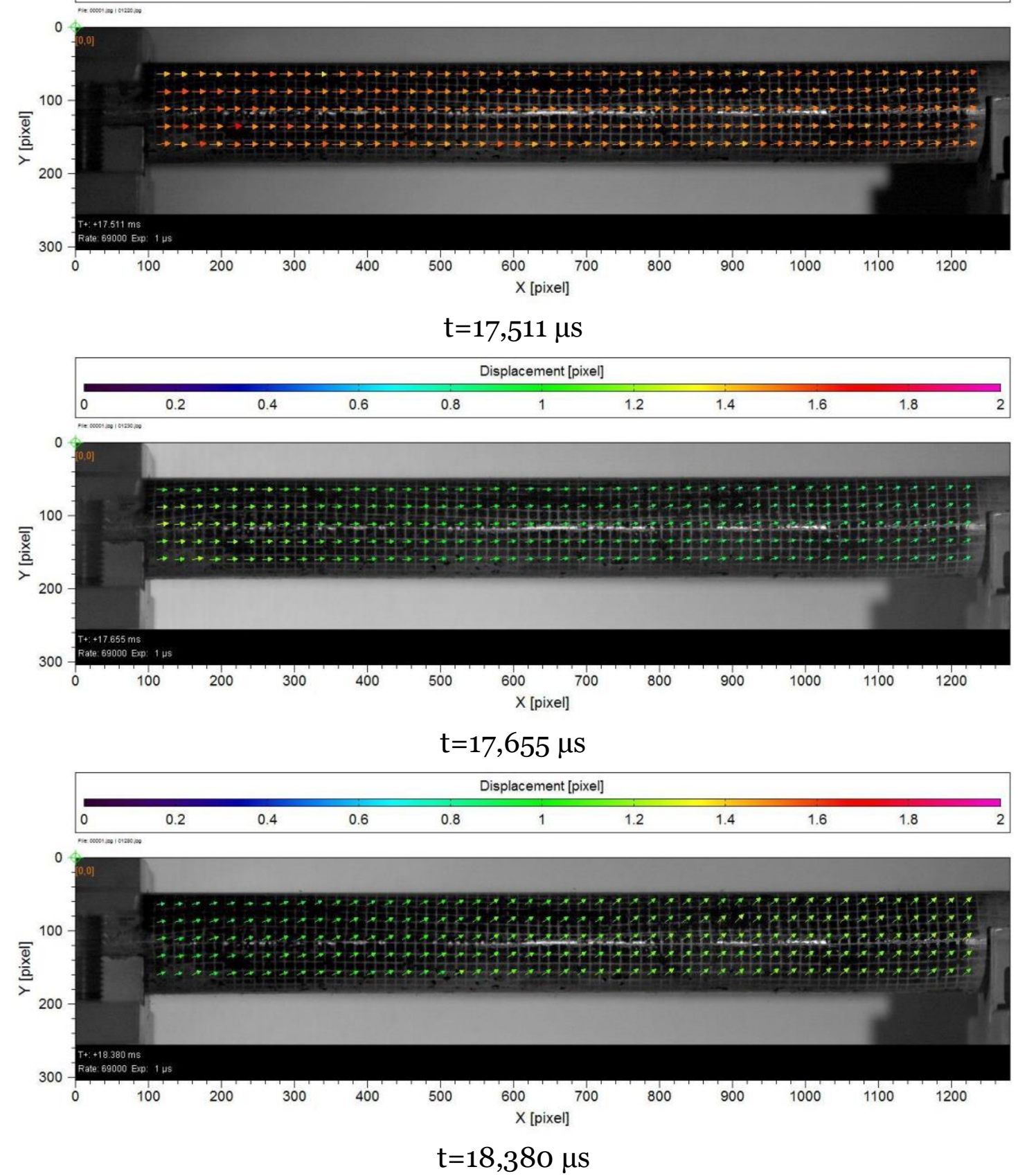

Figure 3 - Fragments of cross-correlation processing.

Three marker points $\mathrm{A}, \mathrm{B}$, and $\mathrm{C}$ were selected to determine the space-time dependences of the oscillations of the elements of the recorded surface (Figure 4). Based on the results of cross-correlation processing, the dynamics of the local movement of the $\mathrm{BB}$ surface at the selected points is estimated. The obtained time dependences of the movement of the BB surface in the coordinate system associated with the selected marker point are shown in Figure 5. 


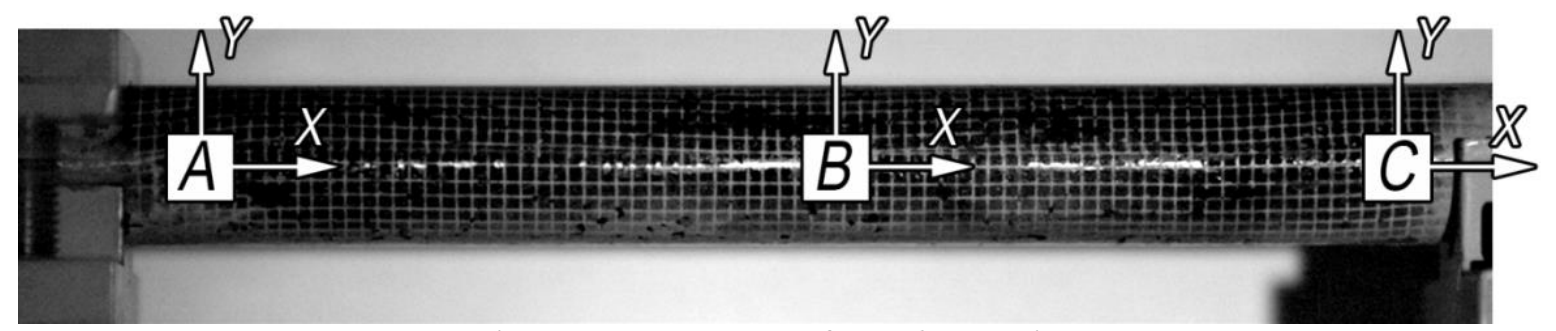

Figure 4 - Layout of marker points.
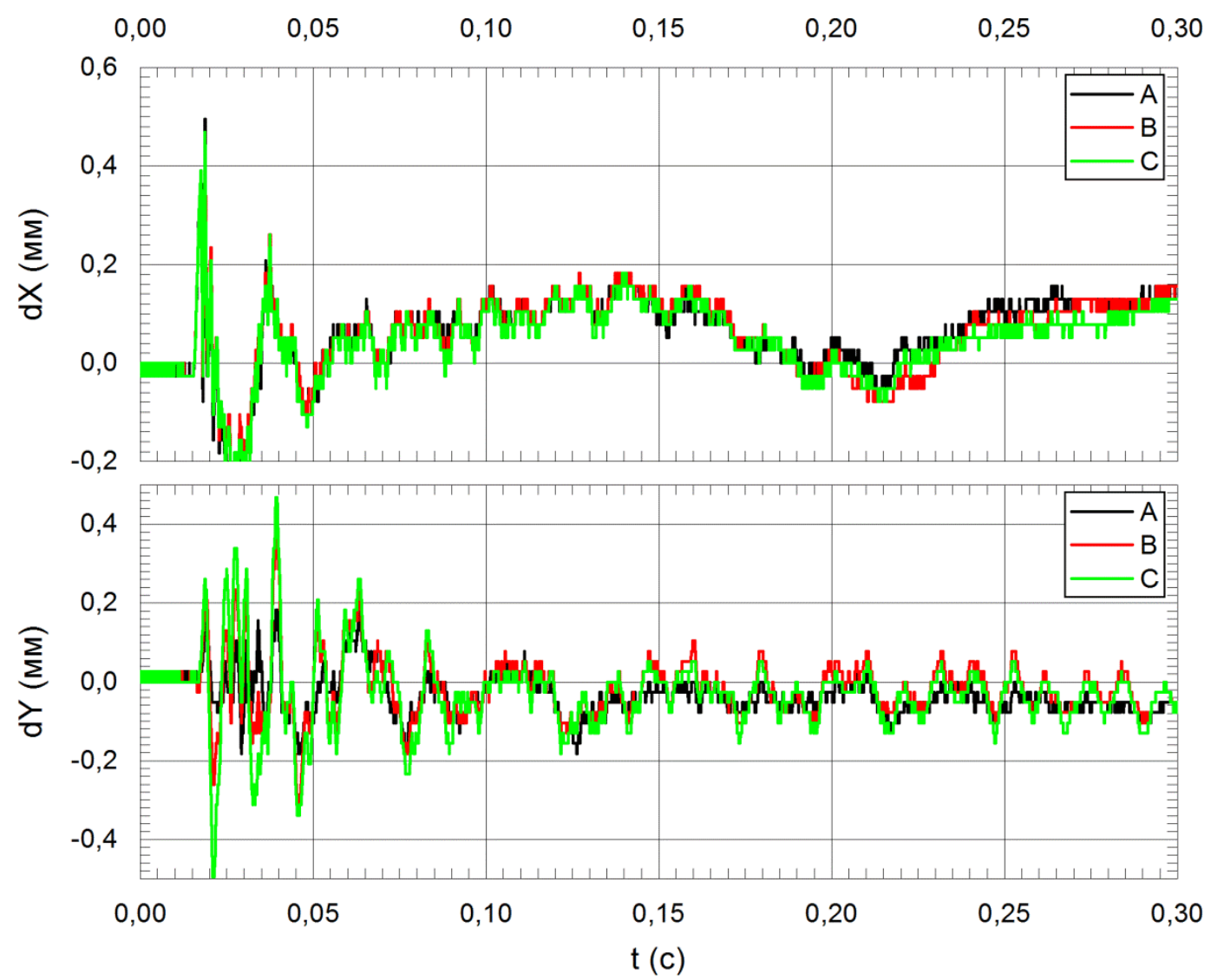

Figure 5 - Dynamics of local movement of the surface of the barrel of a ballistic missile.

The largest amplitude value of the displacement was registered at the initial stage and was $5 \mathrm{~mm}$ in the horizontal and $0.5 \mathrm{~mm}$ in the vertical directions. An enlarged fragment of the obtained dependences, in comparison with the registered values of the pressure in the charging chamber, is shown in Figure 6. 


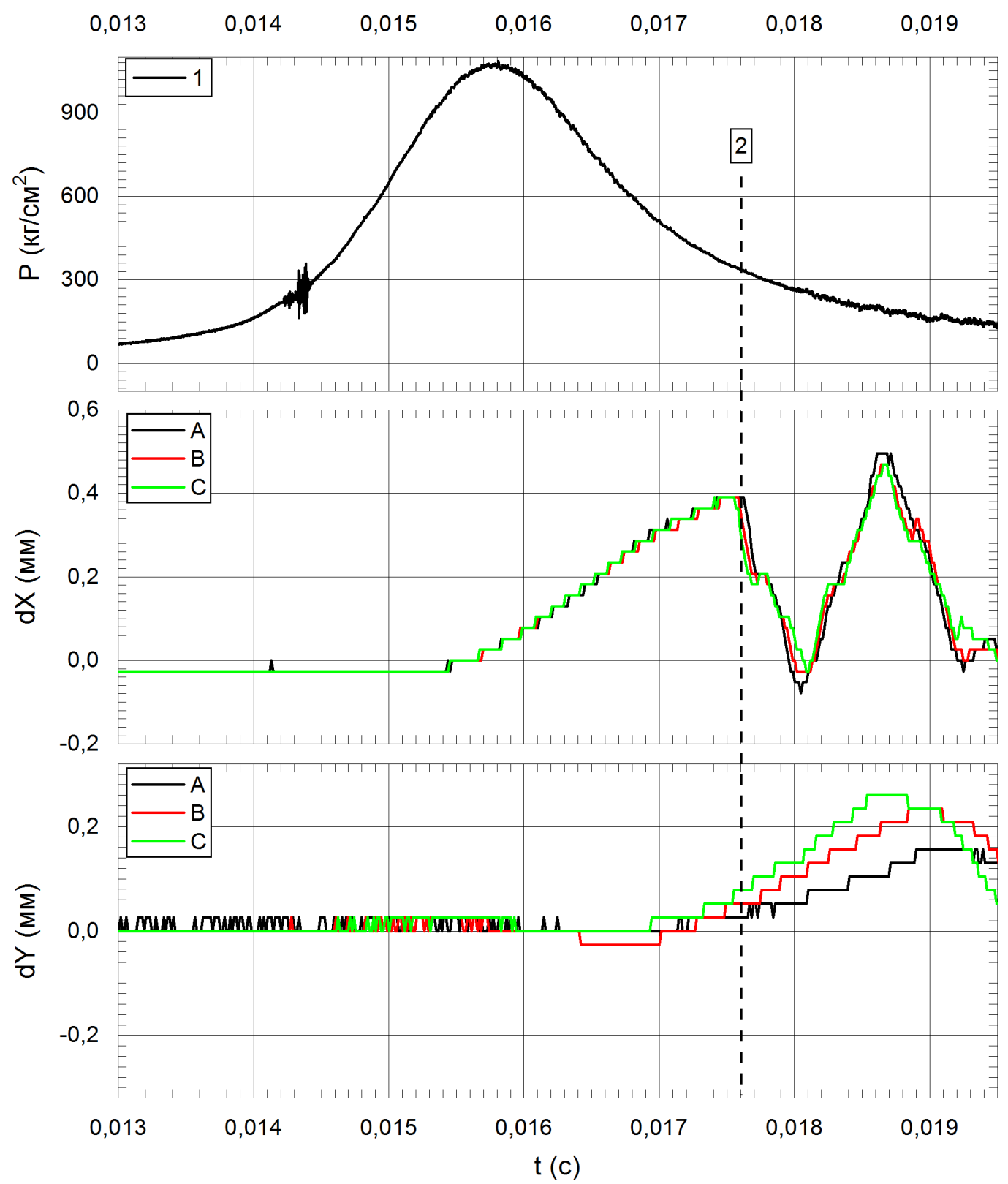

Figure 6 - The initial stage of the dynamics of local displacement (A, B, C), the pressure in the charging chamber (1) and the moment of appearance of the muzzle exhaust (2).

As the pressure in the charging chamber increases, the $\mathrm{BB}$ starts to move in the direction opposite to the movement of the striker. With the formation of the muzzle exhaust, the direction of movement of the $\mathrm{BB}$ in the horizontal plane changes. In the vertical direction, a consistent increase in the displacement amplitude at points C-B-A. Against the background of the BB displacements, the deformation field, which has lower absolute values of displacements, could not be visualized using the presented approach. In this regard, the array of images was re-processed by the cross-correlation algorithm (the second stage of processing) by analyzing consecutive pairs of images of the BB. A fragment of the processing results is shown in Figure 7. 


\begin{tabular}{|llllllll}
\hline \multicolumn{10}{|c|}{ Displacement [pixel] } \\
\hline 0 & 0.025 & 0.05 & 0.075 & 0.1 & 0.125 & 0.15 & 0.175 \\
\hline
\end{tabular}
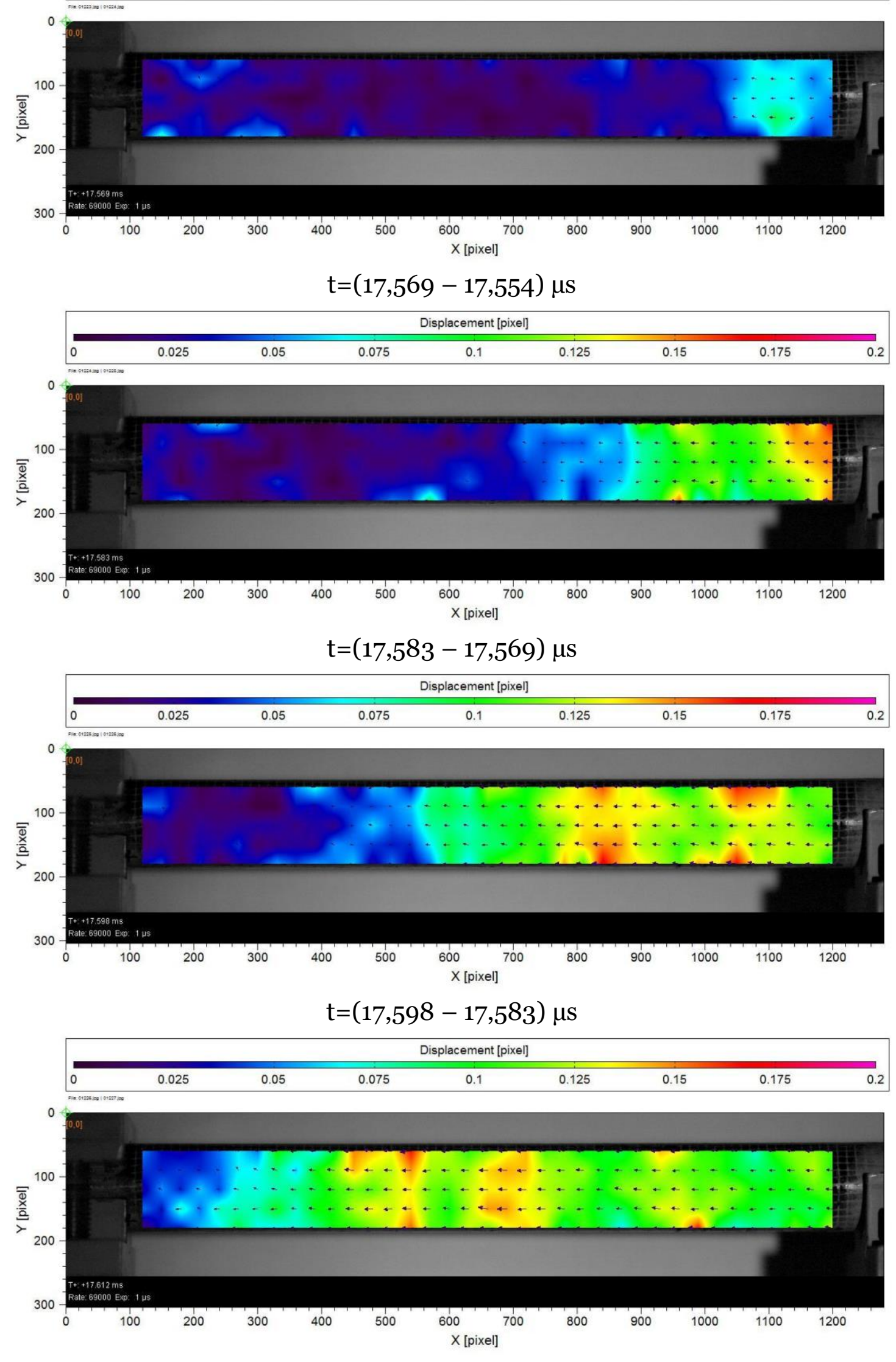

$\mathrm{t}=(17,612-17,598) \mu \mathrm{s}$

Figure 7 - Fragments of cross-correlation processing between consecutive frames. 
The strain field of the BB is visualized. The boundary of the field is shifted in the direction of the striker's movement. The diagram of the displacement of the boundary of the strain field along the BB relative to its muzzle section is shown in Figure 8 . In the experiment, the position of the striker after exiting the barrel at a distance of $488 \mathrm{~mm}$ and at a time of $17.72 \mathrm{~ms}$ relative to the launch of the LGG-5.6 was recorded using an X-ray device (Figure 9).

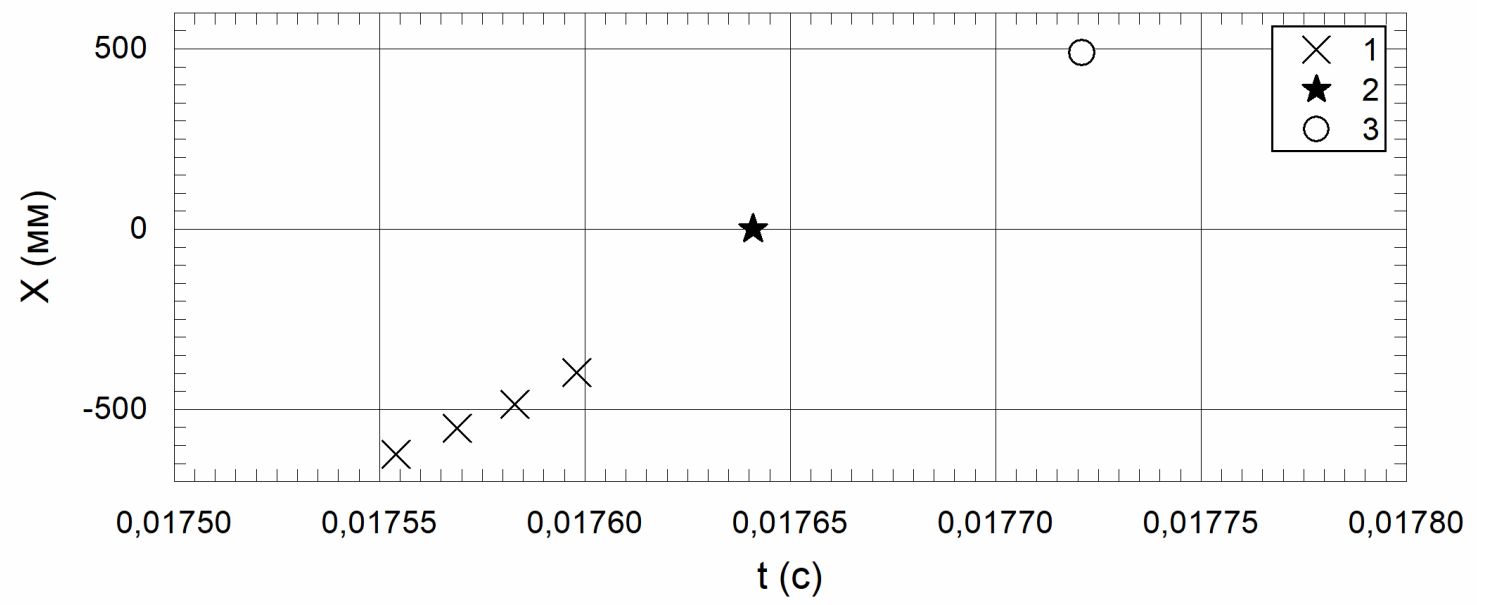

Figure 8-Diagram of the displacement of the boundary of the deformation field (1) in comparison with the information about the muzzle exhaust (2) and the position of the striker in flight (3).

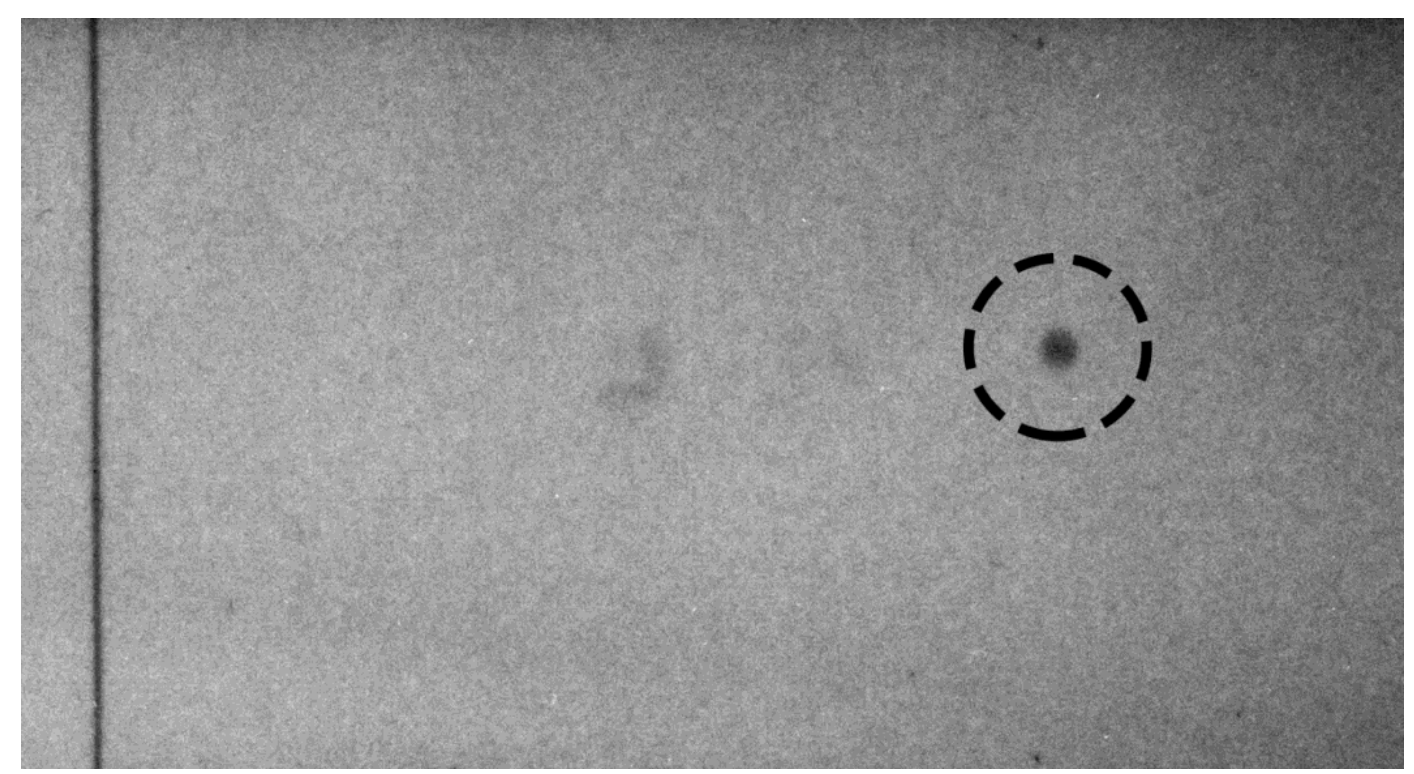

Figure 9-X-ray image of the striker in flight.

The analysis of the obtained data together with the information about the time of formation of the muzzle exhaust allows us to conclude that the registered deformation field corresponds to the passage of the impactor through the BB channel.

\section{Conclusion}

The use of high-speed video recording of the cylindrical shell surface and subsequent cross-correlation analysis of the obtained images allow us to determine the spatial and temporal dependences of the movement of the recorded surface, and to visualize the field of deformations. This possibility is demonstrated by the example of high-speed video recording of a fragment of the barrel of a light-gas gun LGG-5,6 in the process of firing. 
Vibrations of the barrel in the vertical and horizontal directions were recorded, the maximum amplitude value of which was $5 \mathrm{~mm}$. The field of longitudinal elastic deformations of the barrel caused by the passage of the striker through the barrel channel is visualized. The spatial resolution of the registration scheme was 3.84032 pixels / $\mathrm{mm}$, which provided registration (visualization) of displacements up to $0.03 \mathrm{~mm}$.

The work was carried out with the financial support of the RNF (grant 20-19-00613)

\section{References}

1. Adrian, R.J. Twenty years of particle image velocimetry. // Experiments in Fluids. 2005. Vol. 39. No. 2. P. 159-169.

2. Znamenskaya, I.A. Methods of digital analysis of images of liquid and gas-plasma flows based on cross-correlation processing / I.A. Znamenskaya, E.Yu. Koroteeva, F.N. Glazyrin // Scientific Visualization. - 2018. - Vol. 10. - No. 4. - p. 111-119. - DOI 10.26583/sv. 10. 4. 08 .

3. Yagodnitsyna, A.A. Visualization of the flow in an evaporating drop on a substrate using the micro-PIV method / A.A. Yagodnitsyna, A.V. Bilsky, O.A. Kabov // Scientific visualization. - 2016. - Vol. 8. - No. 2. - p. 53-58.

4. Experimental study of the motion of a striker in a sandy medium by a non-contact method / S. I. Gerasimov, A.V. Zubankov, V.I. Erofeev [et al.] // Applied Mechanics and Technical Physics. - 2021. - Vol. 62. - № 1(365). - PP. 159-164 - DOI 10.15372 / PMTF20210117.

5. Modeling and visualization of an explosion in sandy soil. Calculation and experiment / V.A. Kuzmin, V.A. Kikeev, S.I. Gerasimov [et al.] // Scientific Visualization. 2020.- Vol. 12. - No. 3. - pp. 79-88. - DOI 10.26583/sv. 12. 3. 07.

6. Osintsev, A.V. Visualization of displacements and deformations by the method of digital image correlation / A.V. Osintsev, K.Yu. Points / Scientific Visualization. - 2016. Vol. 8. - No. 2. - p. 15-23.

7. Mineev, S.A. Analysis of speckle images of deformable surfaces based on optical flow processing algorithms / S.A. Mineev, A.Yu. Ugolnikov, L.B. Lozovskaya // Bulletin of the Lobachevsky Nizhny Novgorod University. - 2014. - No. 2-1. - pp. 81-86.

8. Orlov B.V., Larman E.K., Malikov V.G. Device and design of artillery barrels: monograph. Moscow: Mashinostroenie, 1976. $432 \mathrm{p}$.

9. Serebryakov M.E. Internal ballistics of barrel systems and powder rockets. Moscow: Oborongiz, 1962. $705 \mathrm{p}$.

10. Khomenko, Yu.P. Mathematical modeling of intra-ballistic processes in stem systems / Yu. P. Khomenko, A.N. Ishchenko, V.Z. Kasimov; Tomsk State University, Research Institute of Applied Mathematics and Mechanics. - Novosibirsk: Publishing House of the Siberian Branch of the Russian Academy of Sciences, 1999. - 256 p. - ISBN 5769202459 .

11. Kinyaev A.A., Lapichev N.V. Stand for the study of high-speed collisions. // Patent of the Russian Federation for invention No. 2653107. Byul. No. 13, 2018.

12. Morphology of damage to thick-walled witness plates by fragments of high-speed strikers made of various materials / P.N. Kalmykov, N.V. Lapichev, I.A. Mikhailov [et al.]// Mechanics of composite materials and Structures. - 2019.- T. 25. - No. 1. - P. 37-56.

13. Visualization of the muzzle exhaust when fired from a light-gas gun / S. Gerasimov, V. Erofeev, I. Kanygin [et al.] // Scientific visualization. - 2014. - Vol. 6. - No. 2. - P.92-103.

14. PIVview: user manual / PIVTEC: web-site of the PIVTEC GmbH (Particles, Imaging and Velocimetry). Gottingen, Germany. 2021. URL: http://www.pivtec.com. 